Revista Digital Universitaria

Vol. 22, Núm. 6, noviembre-diciembre 2021

\title{
Una red neuronal para la detección de somnolencia en conductores
}

\author{
Edurnet Jhaquelin Luna Becerril, Emmanuel Tonatihu Juárez \\ Velázquez y Adolfo Meléndez Ramírez
}

\begin{abstract}
Resumen
En el presente artículo se muestra el uso de una técnica de inteligencia artificial denominada machine learning, para detectar los estados de somnolencia y de vigilia en los conductores vehiculares. La somnolencia se genera cuando el individuo está con un alto nivel de estrés o cansancio, lo cual puede provocar un accidente; cuando está en alerta, se le denomina estado de vigilia. Para su medición, se toman en cuenta dos variables: el pulso cardíaco y la temperatura corporal. El pulso cardíaco se midió con un sensor, a través del método de fotopletismografía (PPG). La temperatura corporal se tomó mediante un sensor infrarrojo, sin contacto. Los datos obtenidos a través de la lectura sirvieron para crear una colección de datos y realizar el entrenamiento de una red neuronal con aprendizaje supervisado. Posteriormente, la información fue clasificada mediante este sistema de adquisición de datos.
\end{abstract}

Palabras clave: machine learning, red neuronal, sensores, somnolencia, vigilia.

\section{A NEURAL NETWORK FOR THE DETECTION OF DROWSINESS IN DRIVERS}

\begin{abstract}
The following article shows the use of machine learning, a type of artificial intelligence, to detect somnolence and the state of vigil in drivers. Somnolence is generated when an individual has high levels of stress or tiredness, and can lead to a potential accident. When alert, it is called vigil state. Two variables are considered: heartbeat and body temperature. Heartbeat was measured with a sensor through the photopletismograph method (PPG). Body temperature was taken through an infrared sensor without contact. The obtained data was used to create a database and to train a neuron network with supervised learning. Later, the information was classified through this data acquisition system.
\end{abstract}

Keywords: machine learning, neural network, sensors, somnolence, vigil. 
Edurnet Jhaquelin Luna Becerril edurnet.luna@gmail.com orcid.org/0000-0003-3435-263X

Docente de la Universidad Politécnica de Texcoco en las carreras de Sistemas Computacionales, Electrónica y Telecomunicaciones. Es alumna de maestría del Tecnológico de Estudios Superiores de Ecatepec. Sus áreas de interés son machine learning, sistemas embebidos y análisis de datos.

Emmanuel Tonatihu Juárez Velázquez

ejuarezv@hotmail.com

Doctor en Comunicaciones y Electrónica por el Instituto Politécnico Nacional. Actualmente se desempeña como profesor e investigador en el Tecnológico Nacional de México y profesor de posgrado en diferentes instituciones educativas. Entre sus áreas principales de experiencia se encuentran las redes de computadoras, los sistemas operativos, los sistemas embebidos, la seguridad informática y los sistemas electrónicos.

\section{Adolfo Meléndez Ramírez}

adolfo_melendez@tese.edu.mx orcid.org/0000-0002-4751-0089

Doctor en Sistemas Computacionales y docente Investigador en el Tecnológico de Estudios Superiores de Ecatepec, en la división de Ingeniería en Sistemas Computacionales. Sus áreas de investigación son seguridad informática, fuzzy systems y machine learning. 


\section{Introducción}

L

a Comisión Nacional de Seguridad de México (2013) reportó que entre 2016 y 2017 hubo un alto índice de accidentes automovilísticos, de los cuales 90\% fue ocasionado por individuos que conducían bajo los efectos del alcohol, a exceso de velocidad y/o en estado de fatiga. De estos accidentes, $30 \%$ fue derivado del estado de somnolencia, debido a la falta de sueño. Al respecto, se conoce que alrededor de $75 \%$ de los conductores ha sufrido al menos un episodio de sueño mientras conduce (Lanuza Moreno et al., 2018).

El descanso y el sueño son aspectos fundamentales para mantener la salud física, pues el gasto de energía de los seres humanos se repone durante el sueño, lo que permite un funcionamiento óptimo del organismo (Basco et al., 2010). Si el cuerpo no descansa adecuadamente llega a tener episodios de sueño y se genera somnolencia. Esto se debe al modelo de los estados del sueño y vigilia, que determina la interacción entre el estar dormido y el estar despierto (Rosales Mayor, 2010). Cada una de estas etapas tiene distintos componentes que se derivan de la actividad de las neuronas en el sistema nervioso, influenciada por los ritmos circadianos, ${ }^{1}$ así como los efectos de factores homeostáticos, ambientales.

La somnolencia es un desequilibrio o alteración, y es algo difícil de medir, ya que está determinada por la calidad y cantidad del sueño, y el ritmo circadiano. Las personas con menor descanso presentan reducciones o incrementos en los ritmos circadianos, y son afectados por variables biológicas, como la temperatura corporal, pulso cardíaco, tensión arterial, entre otros. Cuando un individuo no descansa correctamente y se encuentra despierto o soñoliento, ejerce una acción en la que necesita estar alerta, en la que su ritmo circadiano y sus variables biológicas están por debajo de lo normal. En cambio, cuando la persona tiene un ritmo circadiano alto, es porque sus niveles biológicos están en el rango normal de activación y de alerta —un estado llamado vigilia - , debido a que obtuvo un descanso correcto.

\section{El sueño}

La principal función del sueño es la recuperación fisiológica y psicológica. Las teorías sugieren que el sueño es un proceso activo de múltiples fases. Distintitas ondas cerebrales y actividad del musculo y de los ojos se asocian con las diferentes fases del sueño. (E. 2019).

El sueño normal consta de dos fases:

- Sueño de no movimiento rápido de los ojos (NREM).

- Sueño de movimiento rápido de los ojos (REM).
${ }^{1}$ Son procesos biológicos que se repiten en ciclos de aproximadamente veinticuatro horas. (Sánchez, 2009). 
Figura 1. Fases del sueño (autoría propia) con información de Potter, et al., 2019

\section{El pulso cardíaco}

La frecuencia cardíaca — también llamada ritmo o pulso cardíaca-es la cantidad de veces que el corazón late por minuto. Existen formas de medir dicha latencia, como la pletismografía y la fotopletismografía. ${ }^{3}$

Para la detección del pulso cardíaco en estado de vigilia, se tomó el rango de normalidad entre 60 y 100 pulsaciones por minuto (BPM, por sus siglas en inglés; Tintín et al., 2015), esto según la edad de los usuarios. Debido a lo mencionado, en el estado de somnolencia la frecuencia cardíaca tiende a disminuir 50\%, es decir, que el usuario puede presentar un número de pulsaciones menor a 60 BPм.

\section{Temperatura}

De acuerdo con lo estipulado dentro de los cambios fisiológicos durante el sueño, existe un desequilibrio que indica que la frecuencia cardíaca disminuye en el paso de la vigilia al sueño en la fase NREM $^{4}$ (Navarrete, 2013). Durante esta etapa también disminuye la temperatura corporal. Normalmente, ésta oscila entre 36 a $37.5^{\circ} \mathrm{C}$, y durante el sueño tiene variaciones circadianas y se reduce de 1 a $2{ }^{\circ} \mathrm{C}$. Esto se debe a una mayor sudoración y menor producción de calor (Amador Cano, 2001).

Es una técnica que registra de manera no invasiva las variaciones del volumen sanguíneo en las diferentes extremidades del cuerpo humano (Celi et al., 2011).

${ }^{3}$ Consiste en obtener la señal de las variaciones al aplicar un haz de luz en los dedos.

${ }^{4}$ Sueño de no movimiento rápido de los ojos (E. 2019).

Figura 1.

\section{Aprendizaje supervisado}

Es identificar un aprendizaje a través de un conjunto de datos y un objetivo, en donde los datos son registros almacenados, por ejemplo, la lectura de datos a través de los sensores que se obtuvieron de un grupo de personas en diferentes horarios, como la mañana, tarde-noche y el objetivo es realizar un algoritmo que 
permita descubrir la relación que existe entre las variables de entrada de los datos y unas variables de salida llamadas etiquetas que indican el valor de la predicción a definir, es decir el aprendizaje surge en enseñarle a estos algoritmos cual es resultado que quieres obtener para un determinado valor, tras mostrarle los ejemplos de los datos de entrada, y conocer el valor o etiqueta a identificar, donde el fin es llegar a predecir las etiquetas de nuevos registros que no fueron establecidos, en este campo se hizo uso del tipo de aprendizaje por clasificación donde las lecturas de los datos se seleccionan categóricamente, para conocer los valores posibles, cabe mencionar que los datos no solo realizan el entramiento del aprendizaje, también se requiere de saber su ajuste en una validación, donde los parámetros son el número de neuronas que definen la arquitectura de la red, identificadas como capas y por último la evaluación, para conocer el desempeño del modelo sobre los datos.

Video. Aprendizaje supervisado (Diplomatura en Ciencia de Datos, 2020)

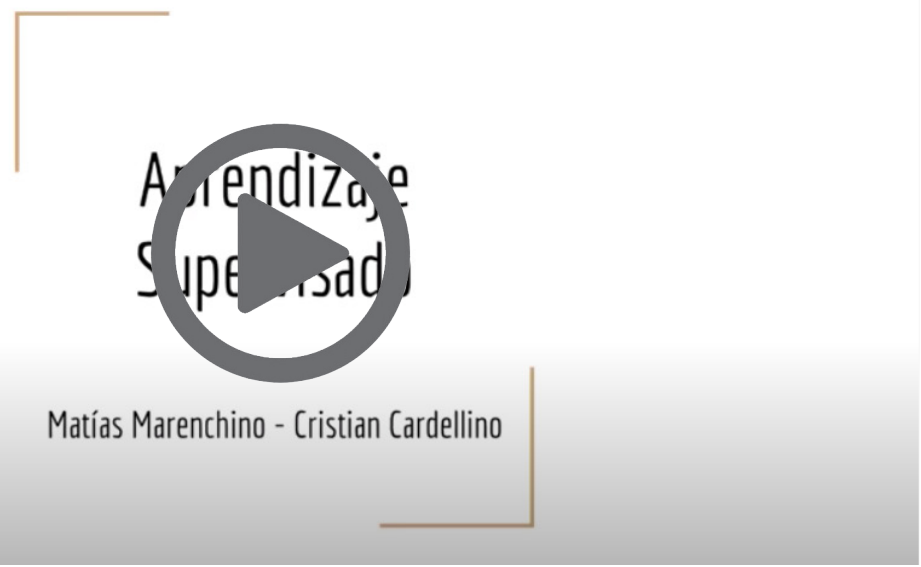

\section{Lectura de datos}

Los datos obtenidos para el pulso cardiaco fueron extraídos por el sensor que identifica la técnica fotopletismografía y para la temperatura se eligió el sensor que detecta lecturas mediante una luz infrarroja, sin tener contacto físico con la superficie. Para realizar las lecturas se requiere de la calibración de los sensores conectados a un microcontrolador programado de acuerdo con los rangos normativos de cada una de las técnicas.

La lectura de los datos se realizó a diversos tipos de personas, las cuales se eligieron en diferentes horarios para indicar el ritmo circadiano y conocer los niveles fisiológicos y determinar si se encontraban en un estado vigilia o soñolientos (somnolencia). Los datos extraídos de los sensores a través del microcontrolador registran lecturas cada 10 segundos, en la figura 3 se observa el monitoreo gráfico de las lecturas de los datos. 
Figura 2. Lectura de los datos (autoría propia).

Figura 3. Mapa del proceso de entrenamiento (autoría propia).

${ }^{5}$ La señal de entrada llamada bias es un valor binario $(0,1)$ de sesgo que permite cambiar o disparar la función de activación

hacia la izquierda o derecha para garantizar un aprendizaje exitoso. (Pt. I. 2019).
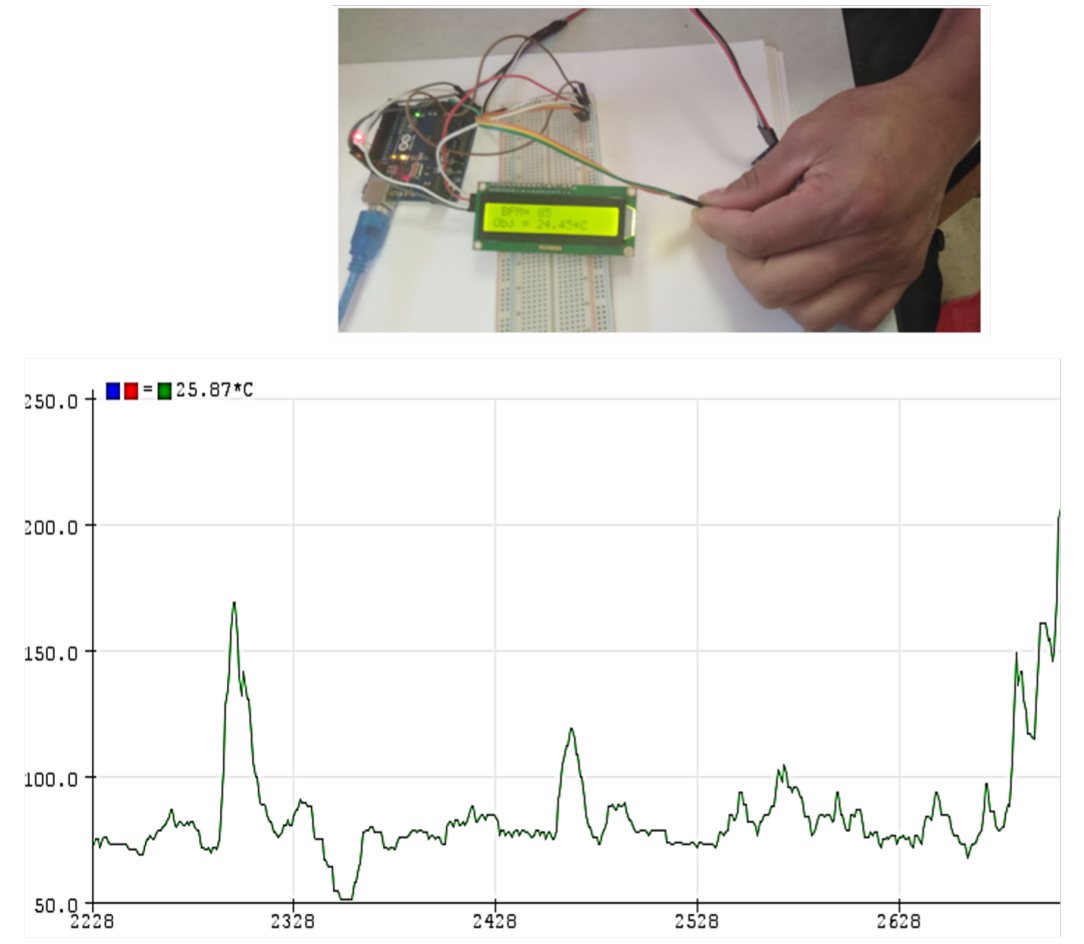

Entrenamiento de los datos

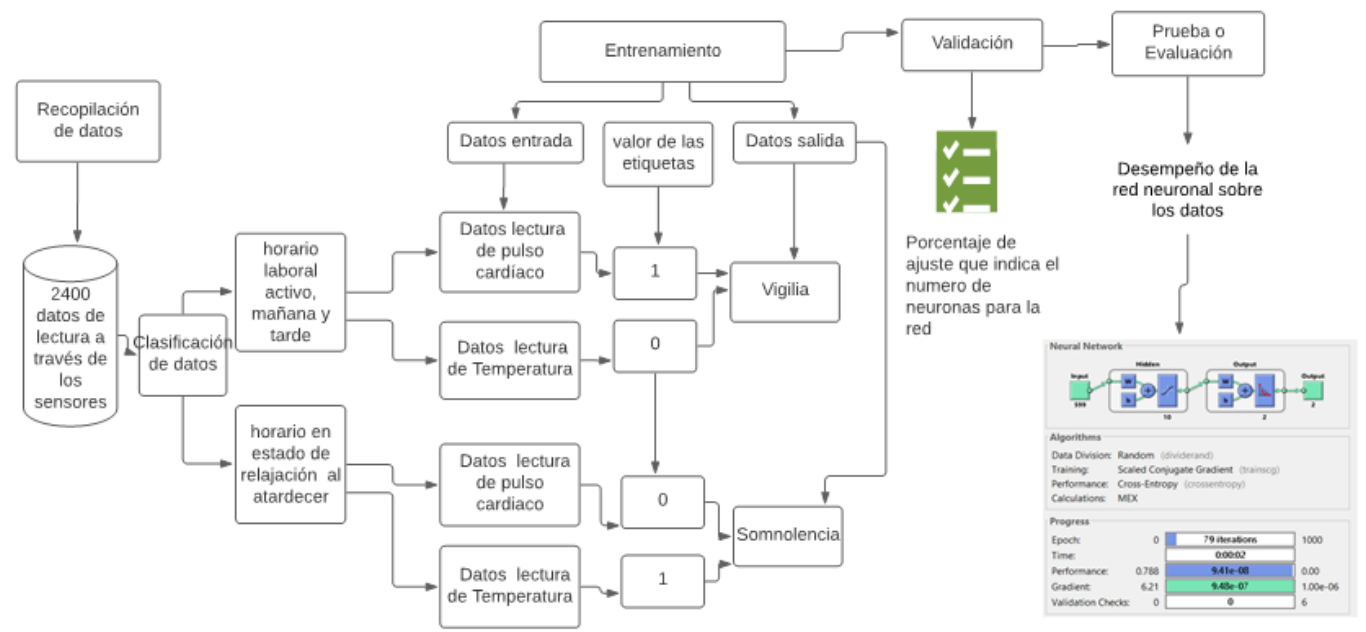

\section{Resultados}

El entrenamiento que realizamos realizó que las lecturas de los sensores fueran clasificadas de manera correcta, con un resultado altamente favorable, al identificar las bias ${ }^{5}$ de forma adecuada para la red neuronal. Es necesario indicar que no se logró generar un buen resultado en las primeras iteraciones 
del entrenamiento, pues se tuvieron que realizar 79 intentos y elevar el número de neuronas a 10 capas ocultas, con el fin de lograr 100\% de entrenamiento, con un margen de error de 0\%.

De esta forma, las variables fisiológicas como el pulso cardíaco (BPM) y la temperatura corporal pueden ser de gran ayuda para la detección del estado de somnolencia y vigilia en los conductores. No obstante, debido a las veces que se tuvo que realizar el entrenamiento, durante el ritmo circadiano se generó una discrepancia en los valores de entrada. En este punto se tiene que tomar en cuenta que los datos deben de ser más precisos, es decir, con mediciones de pulsaciones cardiacas y temperatura casi perfectas de acuerdo con el retiro de la matriz de confusión.

\section{Conclusiones}

A partir de la medición de pulso cardíaco y temperatura corporal, y mediante un modelo de aprendizaje supervisado, se puede observar una convergencia con los estados de salida según la red neuronal aplicada. Por ello, se determina que el estado de somnolencia de un conductor se puede medir con dicho modelo y variables fisiológicas, en un tiempo de 10 segundos y ser analizadas en menos de 5 segundos.

\section{Referencias}

* Aguirre Navarrete, R. I. (2013). Cambios Fisiológicos en el Sueño. Revista Ecuatoriana de Neurología, 22(1-3). https://cutt.ly/uWhhnwe

* Amador Cano, G. (2001). Termorregulación de juveniles y adultos de la langosta de quelas rojas Cherax quadricarinatus [Tesis de Maestría, Centro de Investigación Científica y de Educación Superior de Ensenada]. https://cutt.ly/iWhhthe

* Baca Flores, P. E. y Bonilla Sánchez, K. A. (2017). Prototipo de un sistema de medición de temperatura corporal y pulso cardiaco, para registro médico digitalizado, en la clínica universitaria de becados internos de la unan-Managua [Tesis de Ingeniería, Universidad Nacional Autónoma de Nicaragua]. https://repositorio.unan.edu. ni/9845/1/98913.pdf

- Basco Prado, L., Fariñas Rodríguez, S. e Hidalgo Blanco, M. Á. (2010). Características del sueño de los pacientes en una unidad de cuidados intensivos. Revista Cubana de Enfermería, 26(2). https://cutt.ly/VWhhVc0

* Berzal, F. (2015). Clasificación y predicción. DECSAI.

* Celi, G., Rocha, M. y Yapur, M. (2011, 13 de septiembre). Mediciones fotopletismográficas. Facultad de Ingeniería en Electricidad y Computación [Artículo de Tesis de Grado, Escuela Superior Politécnica del Litoral]. http://www.dspace. espol.edu.ec/handle/123456789/17030 
* Comisión Nacional de Seguridad. (2013). Accidentes y sus factores. https://cutt.ly/HWhkzoh

* E. (2019, 27 septiembre). Las fases del sueño: NREM Y REM. Ambiente idóneo y beneficios para la salud. Elsevier Connect. https://www.elsevier.com/es-es/ connect/enfermeria/las-fases-del-sueno-nrem-y-rem

* Introducción a las Redes Neuronales Pt. I. (2019, 25 junio). Future Lab. https:// futurelab.mx/redes\%20neuronales/inteligencia\%20artificial/2019/06/25/intro-aredes-neuronales-pt-1/

* Lanuza Moreno, S. S. y Lira Dávila, K. E. (2018). Condiciones de trabajo, salud y perfil de accidentabilidad en los taxistas de la ciudad de León, en el período 2017-2018 [Tesis Médico y Cirujano, Universidad Nacional Autónoma de Nicaragua, León]. https://cutt.ly/vRqqDWP

* LeCun, Y., Bengio, Y. y Hinton, G. (2015, mayo). Deep learning. Nature, 521(7553), 436-444. https://doi.org/10.1038/nature14539

* Márquez Mamano, E. A. (2011). Medidor de frecuencia cardíaca y temperatura corporal [Tesis de Licenciatura, Universidad Mayor de San Andrés]. Repositorio institucional. https://cutt.ly/SWhj7Zp

* Matich, D. J. (2001). Redes Neuronales: Conceptos básicos y aplicaciones. Universidad Tecnológica Nacional - Facultad Regional Rosario. https://cutt.ly/VWhjzO8

* Petzold, C. (2008). The annotated Turing: a guided four through Alan Turing's historic paper on computability and Turing machine. Wiley Publishing.

* Potter, P. A., Perry, A. G., \& Stockert, P. A. (Eds.). (2019). Fundamentos de enfermería. Elsevier Health Sciences.

* Rosales Mayor, E. y Rey de Castro Mujica, J. (2010). Somnolencia: Qué es, qué la causa y cómo se mide. Acta Médica Peruana, 27(2), 137-143. http://www.scielo.org. pe/pdf/amp/v27n2/a10v27n2.pdf

* Saavedra Torres, J. S., Zúñiga Cerón, L. F., Navia Amézquita, C. A. y Vásquez López, J. A. (2013). Ritmo circadiano: el reloj maestro. Alteraciones que comprometen el estado de sueño y vigilia en el área de la salud. Morfolia, 5(3). https://revistas.unal. edu.co/index.php/morfolia/article/view/41615

* Sánchez, S. C. A. (2009). Los ritmos circadianos y la productividad laboral. El Cuaderno Ciencias Estratégicas, 3(5), 39-57.

* Sanz Fernández,J. (1989). Biofeedback de temperatura periférica. Revista Española de Terapia del Comportamiento, 7(3), 273-294. https://eprints.ucm.es/id/eprint/27176/

* Tintín Durán, E. I. (2015). Diseño y elaboración de un prototipo de monitor de signos vitales aplicando métodos no invasivos con comunicación de datos a dispositivos móviles [Tesis de Ingeniería, Universidad Politecnica Salesiana]. https://dspace. ups.edu.ec/bitstream/123456789/7982/1/UPS-CT004847.pdf

* Velayos, J. L., Moleres, F. J., Irujo, A. M., Yllanes, D. y Paternain, B. (2007). Bases anatómicas del sueño. Anales del sistema sanitario de Navarra, 30(7-17). https:// scielo.isciii.es/pdf/asisna/v30s1/02.pdf 


\section{Cómo CITAR ESTE ARTículo}

* Luna Becerril, Edurnet Jhaquelin, Juárez Velázquez, Emmanuel Tonatihu y Meléndez Ramírez, Adolfo. (2021, noviembre-diciembre). Una red neuronal para la detección de somnolencia en conductores. Revista Digital Universitaria (RDU), 22(6). http://doi.org/10.22201/cuaieed.16076079e.2021.22.6.1 